\title{
STORTHYNGURA MAGNIFICA SP. N., ISOPODE ABYSSAL DE L'ATLANTIQUE NORD ${ }^{1}$ )
}

\author{
PAR \\ PIERRE CHARDY \\ Centre Océanologique de Bretagne, B.P. 337, 29273 Brest, France
}

- Le genre Storthyngura Vanhöffen, 1914, largement représenté à l'échelle de l'océan mondial, compte 5 espèces en Atlantique nord: S. atlantica Beddard, 1885; S. truncata Richardson, 1908; S. magnispinis Richardson, 1908; S. vemae Menzies, 1962 et S. shanoi Menzies, 1962. Lors de la campagne Noratlante du N.O. „Jean Charcot" (août - septembre 1969) nous avons récolté une nouvelle espèce de ce genre dont la description fait l'objet de ce travail.

Storthyngura magnifica sp. n, (figs. 1 et 2)

Matériel étudié. - Campagne Noratlante (août - octobre 1969). Station 2, prélèvement E 01 $\left(53^{\circ} 54,9^{\prime} \mathrm{N} 17^{\circ} 51,8^{\prime} \mathrm{W}\right)$. Profondeur: $2456 \mathrm{~m}$. Un seul exemplaire $\hat{\delta}$, déposé au Muséum national d'Histoire naturelle de Paris. L'engin de prélèvement est une drague épibenthique inspirée de I'"epibenthic sled" de Hessler \& Sanders (1967).

Description. - L'holotype est un mâle adulte mesurant $6 \mathrm{~mm}$ de l'extrémité du rostre au bord postérieur du pléotelson. La forme générale du corps est allongée, caractérisée par les prolongements latéraux des somites épineux (fig. 1A).

Céphalon quadrangulaire, plus large que le péréionite 1 (abstraction faite des épines coxales). Présence d'une paire d'épines dorsales bien développées, légèrement recourbées vers l'intérieur.

Péréion. Péréionite 1 dépourvu d'ornementation dorsale, et prolongé latéralement par un processus coxal muni d'une simple épine. Péréionites 2-4 porteurs de 3 courtes épines sur la marge antéro-dorsale et prolongés latéralement par un processus coxal à deux épines. Péréionite 4 muni d'une longue épine antéro-latérale. Péréionites 5-7 coalescents, armés chacun de deux fortes épines dorsales, et prolongés latéralement par un processus spiniforme dont la longueur est décroissante du sème au 7 ème segment.

Pléotelson formé d'un seul segment. Bords latéraux armés d'une paire d'épines antérieures, perpendiculaires à l'axe de l'allongement du telson, et d'une paire d'épines postérieures, légèrement dirigées vers l'arrière. Extrémité médiane de l'épine terminale incisée en „V”. Présence de 2 fortes épines médio-dorsales, et

1) Contribution $n^{\circ} 235$ du Département Scientifique du Centre Océanologique de Bretagne. 
PIERRE CHARDY

A
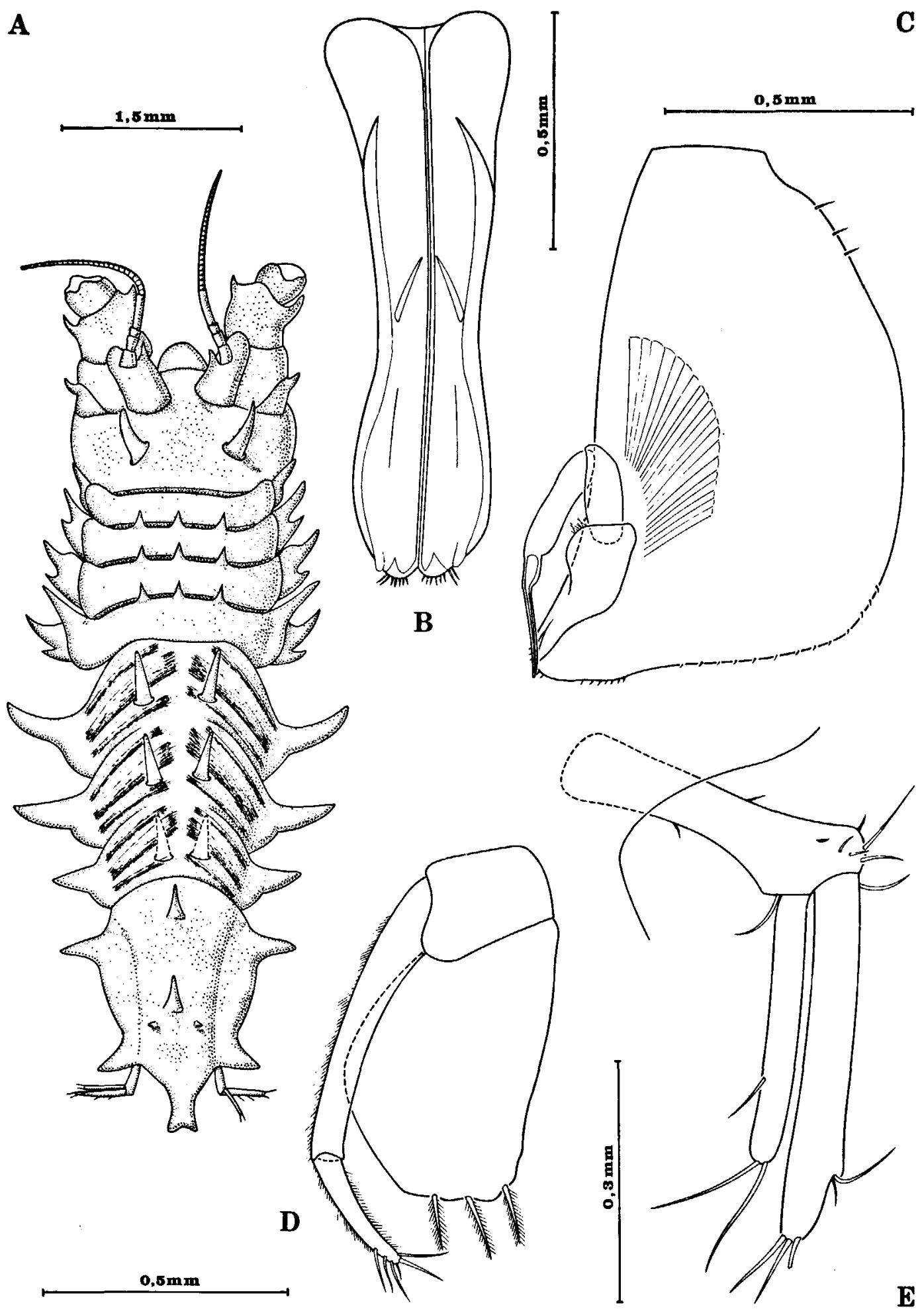

Fig. 1. Storthyngura magnifica sp. n., holotype $\delta$. A, habitus face dorsale; B, première paire de pléopodes $\hat{o} ; \mathrm{C}$, pléopode $2 \hat{o} ; \mathrm{D}$, pléopode $3 ; \mathrm{E}$, uropode. 
STORTHYNGURA MAGNIFICA NOV.

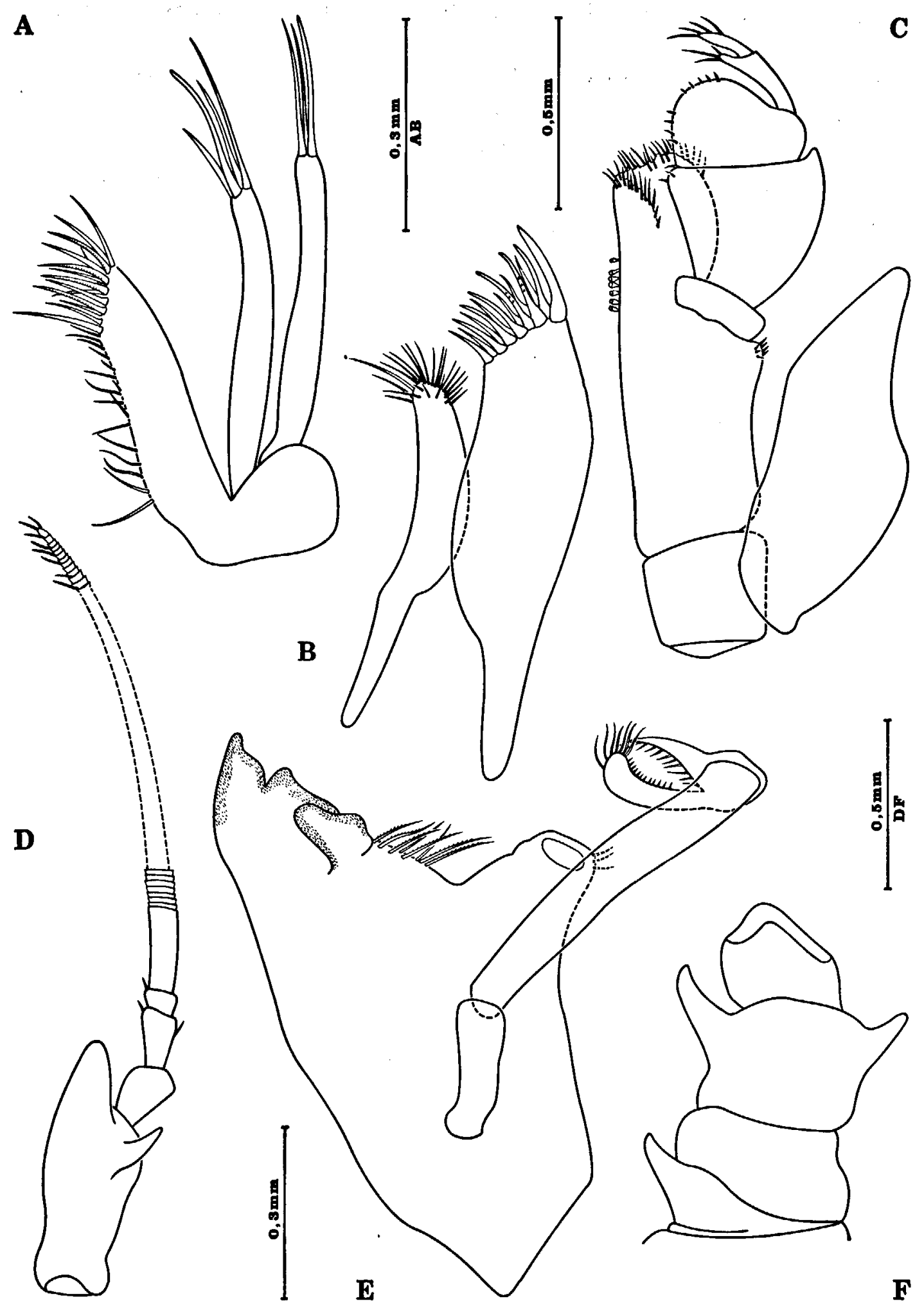

Fig. 2. Storthyngura magnifica sp. n., holotype ô. A, maxille; B, maxillule; C, maxillipède; D, antennule; $\mathrm{E}$, mandibule gauche; $\mathrm{F}$, antenne. 
de 2 tubercules spinescents, situés immédiatement derrière l'épine médio-dorsale postérieure.

Antennule (fig. 2D). Partie distale du premier article pédonculaire développée en un processus conique à la base duquel s'insert une épine interne longue et effilée. Flagellum multiarticulé, comportant une cinquantaine d'articles.

Antenne (fig. 2F) cassée au niveau du 4ème article pédonculaire. Premier segment très court, porteur d'une épine externe. Second segment dépourvu d'épine latérale. Troisième segment armé de 2 épines latérales sur chacun de ses bords.

Mandibule gauche (fig. 2E). Pars incisiva fortement chitinisée, constituée de 2 dents massives et complétée par une lacinia mobilis non denticulée. Pars molaris robuste, de forme conique. Palpe mandibulaire à trois articles, caractéristique du genre Stortbyngura.

Maxille (fig. 2B). Lobe inférieur richement pourvu de soies fines sur la partie distale.

Maxillule (fig. 2A) sans particularité remarquable.

Maxillipède (fig. 2C). Bord interne du basipodite orné de 7 rétinacles. Deuxième et troisième article du palpe fortement élargis en lamelle, conformément à la structure des maxillipèdes chez les Eurycopidae.

Tous les péréiopodes sont cassés au niveau des premiers articles. Leur description fait donc défaut dans cette diagnose.

Pléopode $1 \delta$ (fig. 1B) terminé par un apex arrondi, frangé de quelques soies courtes. Pléopode $2 \hat{o}$ (fig. $1 \mathrm{C}$ ) très élargi muni d'un appareil copulateur réduit terminé par un tube filiforme.

Uropode (fig. 1E). Exopodite presqu'aussi long que l'endopodite.

Discussion et remarques biogéographiques. - Parmi les 38 espèces actuellement connues du genre Storthyngura, 6 d'entre elles sont caractérisées par une incision postéro-médiane du pléotelson: S. elegans Vanhöffen, 1914; S. brachycepbala Birstein, 1957; S. furcata Wolff, 1956; S. serrata Wolff, 1962; S. vemae Menzies, 1962 et S. sepigia George \& Menzies, 1968. Au sein de ce groupe, S. magnifica sp. n. est facilement reconnaissable par l'allongement de l'épine terminale du telson, l'absence d'épine sur le premier segment thoracique et la présence de 3 épines dorsales sur les péréionites 2-4. La combinaison de ces caractères, encore jamais observée chez les représentants du genre Storthyngura, permet de distinguer S. magnifica sp. n. et justifie la création d'une espèce nouvelle.

A la suite de Birstein (1957), et tenant compte des formes nouvellement décrites, George \& Menzies (1968a) ont proposé une classification traduisant les affinités morphologiques des 38 espèces connues du genre Storthyngura. Parmi les 5 groupes et les 14 sous-groupes de cette classification (essentiellement liée à la configuration du pléotelson) $S$. magnifica sp. n. se situe indiscutablement au sein du sous-groupe D 3 dans le groupe D (selon la désignation des auteurs). Les 11 espèces du groupe $\mathrm{D}$ sont caractérisées par le nombre d'épines latérales du pléotelson ( 2 paires) et leur orientation (les épines antérieures sont perpendiculaires à l'axe de symétrie, et les épines postérieures légèrement orientées vers l'arrière). 


\section{STORTHYNGURA MAGNIFICA NOV.}

A l'intérieur du groupe D, George \& Meizies (1968a) distinguent le sous-groupe D 3, réunissant 3 espèces dont l'apex du pléotelson est profondément incisé: S. furcata Wolff, 1956, S. brachycephala Birstein, 1957 et S. sepigia Menzies, 1962. Cet ensemble d'espèces possède une vaste répartition géographique puisqu'il est représenté en Antarctique, dans le Pacifique Nord et Sud, et dans l'Atlantique Sud. S. magnifica sp. n. étend la répartition horizontale de ce groupe à l'Atlantique Nord.

\section{SUMMARY}

Description of a new deep-sea Isopod, Storthyngura magnifica n. sp., collected on the NorthAtlantic abyssal plain. Biogeographical comments.

\section{REFERENCES}

BEDDARD, F. E., 1886. Report on the Isopoda collected by H.M.S. "Challenger" during the years 1873-76, Part 2. Challenger Reps., (Zool.) 17: 1-175.

Birstein, J. A., 1957. Certain peculiarities of the ultra-abyssal fauna at the example of the genus Storthyngura (Crustacea Isopoda Asellota). Zool. Zhurnal, Moscou, 36: 961-985. [En russe, résumé anglais].

George, R. Y. \& R. J. Menzies, 1968. Species of Storthyngura (Isopoda) from the Antarctic with descriptions of six new species. Crustaceana, 14: 275-301.

- \& $1968 \mathrm{a}$. Distribution and probable origin of the species in the deep-sea Isopod genus Storthyngura. Crustaceana, 15: 171-187.

Hessler, R. \& H. Sanders, 1967. Faunal diversity in the deep-sea. Deep-Sea Res., 14: 65-78.

Richardson, H., 1908. Description of a new isopod of the genus Eurycope from Martha's Vineyard. Proc. U. S. nation. Mus., 34: 67-69.

VANhöfFEN, E., 1914. Die Isopoden der deutschen Südpolar Expedition 1900-1903. Deutsche Südpol. Exped., (Zool.) 7 (4): 449-598.

WolfF, T., 1956. Isopoda from depths exceeding 6000 meters. Galathea Reps., 2: 85-157.

- 1962. The systematics and biology of bathyal and abyssal Isopoda Asellota. Galathea Reps., 6: $7-320$. 